\section{¿NUEVOS PATRONES DE INVESTIGACIÓN? DINÁMICAS DE APERTURA Y CIERRE EN EL PROCESO DE INTEGRACIÓN SOCIO-TÉCNICA}

\author{
Andoni Eizagirre \\ Universidad de Mondragón \\ ORCID iD: https://orcid.org/0000-0002-1829-6108 \\ aeizagirre@mondragon.edu
}

\section{NEW RESEARCH PATTERNS? OPEN-AND-CLOSED DYNAMICS IN THE SOCIO-TECHNICAL INTEGRATION PROCESS}

Cómo citar este artículo/Citation: Eizagirre, A. (2019). ¿Nuevos patrones de investigación? Dinámicas de apertura y cierre en el proceso de integración socio-técnica. Arbor, 195 (794): a528. https://doi.org/10.3989/arbor.2019.794n4002

Recibido: 17 julio 2018. Aceptado: 28 marzo 2019.

RESUMEN: La formulación de las políticas de investigación y desarrollo está siendo transformada de manera novedosa en los documentos más recientes que se elaboran en Europa. Una de las peculiaridades es que los patrones de investigación transitan hacia prácticas científico-tecnológicas más interactivas entre los distintos actores de la sociedad. Así, como respuesta a la naturaleza compleja de la investigación las primeras medidas se revelan en aquellas estrategias para la promoción de actitudes emprendedoras y colaborativas entre los actores académico-empresariales de la investigación y el desarrollo. A estas iniciativas se superponen otros planteamientos que trascienden el objetivo primariamente economicista de la colaboración e integran los aspectos sociales y éticos en la investigación y el desarrollo. Sin embargo, estas transformaciones también están sujetas a diversas tensiones de base y a objetivos concurrentes. El artículo dilucida estas dinámicas de apertura-y-cierre y manifiesta que algunas de las relaciones y demandas se muestran más resistentes al cambio.

PALABRAS CLAVE: Política de I+D; competitividad; integración socio-técnica; investigación e innovación responsable; Unión Europea.
Copyright: (C) 2019 CSIC. Este es un artículo de acceso abierto distribuido bajo los términos de la licencia de uso y distribución Creative Commons Reconocimiento 4.0 Internacional (CC BY 4.0).
ABSTRACT: The formulation of research and development policies is being transformed in a novel way in the most recent documents coming out of Europe. One notable peculiarity is that research patterns are moving toward scientific-technological practices that are more interactive among the various actors of society. Thus, the first measures in response to the complex nature of research are being revealed in strategies for the promotion of entrepreneurial and collaborative attitudes among academic-business actors in research and development. Other approaches are superimposed on these initiatives, approaches that transcend the primarily economistic goal of collaboration and that integrate social and ethical factors in research and development. Nevertheless, these transformations are also subject to underlying tensions and concurrent objectives. The article elucidates these opening-and-closing dynamics and demonstrates that some relationships and demands are more resistant to change.

KEYWORDS: R\&D policy; competitiveness; socio-technical integration; responsible research and innovation; European Union. 


\section{INTRODUCCIÓN}

En el campo de las políticas de investigación y desarrollo (I+D) el periodo que discurre entre la posguerra de la Segunda Guerra Mundial y la década de 1980 se consolidó sobre unas peculiares relaciones de la ciencia con la sociedad y desplegó una meta-narrativa fuertemente vinculada a la tradición ilustrada sobre la investigación y la libertad académica (Flink y Kaldewey, 2018). Este legado simbólico comprende la ciencia como un acervo de conocimiento acumulado regido por valores cognitivos y un conjunto de métodos mediante los cuales se certifica el conocimiento genuino (Polanyi, 1951). A ello se suma un principio normativo según el cual la garantía de la buena ciencia, o de la ciencia responsable, es la propia acción del investigador basada en el respeto de las normas científicas (Merton, 1942). De esta manera la responsabilidad de los científicos tutelada por la búsqueda de la verdad y alentada por sus colegas profesionales se erigía como una superación de las responsabilidades más generales hacia la sociedad (Douglas, 2003). Esa idea de que el producto y la finalidad de la investigación son el conocimiento por sí mismo avaló una política de I+D basada en el modelo lineal -i.e,, centrada en la investigación básica, si bien con una finalidad claramente orientada-, que a partir del conocimiento científico interpreta el desarrollo tecnológico y el bienestar social de un modo secuencial y ordenado, y una visión científica del progreso (Steelman, 1948).

Esa serie de arreglos simbólicos e institucionales, que sostuvo la confianza en la autoridad epistémica y social de la ciencia, favoreció -como veremos en el segundo apartado- un planteamiento demarcacionista sobre las relaciones ciencia-sociedad (Sarewitz, 2016). No obstante, en el tercer y cuarto apartado, a través del análisis de los documentos canónicos sobre I+D europeos, sostenemos que, a lo largo de las últimas décadas, de una manera progresiva y acelerada por un conjunto heterogéneo y concurrente de intereses y demandas, mengua la idea de que el propio conocimiento es un fin en sí mismo que encuentra en sí su razón de ser. Por el contrario, los patrones de investigación transitan hacia prácticas científico-tecnológicas más interactivas entre los distintos actores de la sociedad y vienen revisándose los roles preestablecidos. Así, como respuesta a la naturaleza compleja de la investigación y el desarrollo científico-tecnológico, las primeras medidas se revelan en aquellas estrategias para la promoción de actitudes emprendedoras y colaborativas entre los actores académico-empresariales. No obstante, a estas iniciativas se superponen otros planteamientos que trascienden el objetivo primariamente economicista de la colaboración. Por ejemplo, en los Programas Marco para la Investigación en la Unión Europea hay una continua ampliación de áreas de conocimiento -más allá de la genética y la genómica- que integran perspectivas sociales y éticas; se dilata el acervo de asuntos que se estiman pertinentes -más allá de la regulación basada en la evaluación del riesgo- en la gobernanza de la ciencia y la tecnología; se agudiza la relevancia que adquieren esos nuevos actores, perspectivas y asuntos sobre los propios procesos de investigación y constitución del conocimiento.

Este artículo, a través de un análisis histórico y sociológico de la ciencia y de su relación con la sociedad, tiene como propósito comprender esa mayor apertura e integración socio-técnica en las políticas europeas de investigación, lo que también nos servirá para abordar como pregunta de investigación por qué algunas de las relaciones y demandas se muestran más resistentes al cambio. La elucidación de estas tensiones y limitaciones es relevante para entrever las trayectorias que pueden transitar las nuevas políticas europeas de I+D.

\section{CIENCIA Y SOCIEDAD: DIFERENCIACIÓN Y DEMAR- CACIÓN DE ROLES}

Una de las singularidades de las políticas de I+D en el periodo que transcurre entre la posguerra y la década de 1980 es la continuidad del criterio demarcacionista, que asigna y demarca un conjunto de roles a los distintos actores que participan en el proceso social de la actividad científico-tecnológica, pese a las distintas transformaciones en las políticas de estímulo y regulación de la ciencia que se exponen en los siguientes dos subapartados respectivamente.

\subsection{Modalidades de actividad científico-tecnológica más permeables}

Una primera controversia del modelo lineal de I+D centrado en la investigación básica se originó en los Estados Unidos por los resultados desiguales de las misiones orientadas a la investigación contra el cáncer y el programa estratégico del espacio, que en cierta forma generó suspicacias hacia el modelo de la financiación sin límites y a largo plazo de la investigación básica y precomercial. De este modo, en los años 60 se alzaron las primeras voces críticas que cuestionaban la política de patronazgo y reclamaban una actividad científico-tecnológica más proclive a las demandas de mercado y a la transferibilidad del conocimiento generado, es decir, una financiación en aras de los resul- 
tados esperados -más allá de una investigación básica orientada- con un mayor énfasis en la industria y con una preferencia por la ciencia aplicada y la tecnología (Kaldewey y Schauz, 2018).

El periodo de confrontación y coexistencia pacífica característico de la Guerra Fría se distinguió por aunar la escalada de la inversión pública en investigación y proyectos a gran escala y un fuerte vínculo entre la gran ciencia y la defensa nacional. Dicho de otra manera, uno de los principales arreglos económicos de aquel periodo -que explica el financiamiento de proyectos militares con perfil científico- está relacionado con la capacidad simultánea de articular dos categorías políticas, como son por una parte la investigación básica o la fase precompetitiva de la producción del conocimiento científico, y por otra la ciencia orientada y el desarrollo tecnológico, que tienen un carácter determinado por una misión y deben ceñirse a las prioridades fijadas. Aquel arreglo permitió también que en un periodo de fuertes tensiones generadas por la Guerra Fría las políticas de I+D elaboraran un modelo de financiación de la ciencia básica sobre la retórica normativa de la libertad y el bienestar, que legitimó precisamente la investigación en física cuántica, en nuevos materiales y en geociencias en favor de la misión militar (Elzinga, 2012, p. 418).

La reforma del modelo lineal de investigación y una mayor orientación práctica de la investigación y la emergencia conceptual de la transferencia tecnológica resultaron funcionales para proteger la ciencia de cualquier injerencia externa, si bien se acentuó la pérdida relativa de su peso en las políticas de I+D. En este sentido, la división funcional y moral de la actividad científico-tecnológica -en expresión de Rip (2016)- no fue alterada, pese a las carencias analíticas y a los frecuentes reajustes del modelo lineal de investigación, al menos hasta la década de 1980 (Sarewitz, 1996).

A partir de los años 80 los ingredientes básicos que componen las políticas de I+D -i.e. la formulación y articulación de prioridades, las estructuras institucionales, la selección de agentes, el sistema de evaluación y control y los modelos de investigación- se expusieron a una gradual y sucesiva transmutación. Uno de los motivos sustanciales que originaron aquella evolución, que sobresale por una orientación estratégica de la investigación y un énfasis cuasi exclusivo en la innovación tecnológica, fue la baja productividad de las economías occidentales, a la que se adicionaron una mejor comprensión de la naturaleza compleja de la I+D y una globalización multipolar que presionó con unas dinámicas, expec- tativas y alianzas manifiestamente diferentes a las de la Guerra Fría (Jamison, 2012).

Algunos de los rasgos de esa nueva modalidad de actividad científico-tecnológica más permeable por la sociedad y que envuelve un grado mayor de internacionalización son el predominio de criterios economicistas en la justificación, validación, financiación y valoración de los resultados esperados, una tendencia mayor a la medición de la calidad y de la eficacia de la inversión en I+D, y una nueva estructura del sistema de I+D que abarca funciones científicas, tecnológicas, productivas y financieras. El rol de la administración pública se vuelve más activo y sistémico para involucrar las distintas áreas del gobierno y políticas públicas, a la vez que tratará de vincular en calidad, densidad e intensidad a los distintos actores públicos y privados del sistema de I+D (Nelson y Wright, 1992).

No obstante, esta creciente apertura del sistema de I+D a los estímulos económicos -y que moldean precisamente los cinco primeros Programas Marco de Investigación de la Unión Europea entre 1984 y 2002encuentra limitaciones sustanciales para incorporar aquellas demandas de carácter social y ambiental en el modelo de I+D europeo (véase Commission of the European Communities, 2001), hasta el punto de que la respuesta comunitaria a las controversias socio-técnicas y la crisis de confianza de los años 1990 y 2000 vuelve a acentuar la diferenciación entre ciencia y sociedad a través de iniciativas como el fomento de la cultura científica y la integridad de los científicos y la experticia (véase European Commission, 2002). Este tipo de acciones, que parten del modelo de déficit cognitivo (según el cual las controversias sociales ante la tecnología se deben al escaso nivel de conocimiento científico-tecnológico), relegan la sociedad civil al eslabón último de la comercialización y vienen a indicar que las estrategias socio-técnicas integradoras son de tipo fundamentalmente economicista e impermeabilizan las dinámicas expertas de I+D (véase European Commission, 2007).

\subsection{La responsabilidad ampliada de los científicos}

Una dinámica similar de apertura y cierre se condensa en relación con la responsabilidad autorreguladora del científico y su gradual transformación ante la magnitud de las consecuencias adversas ocasionadas por el avance científico-tecnológico (Kleinman, 1995). A lo largo de la segunda mitad del siglo XX las cuestiones metacientíficas sobre la responsabilidad social fueron escrutadas en el seno de la comunidad científica. 
El uso de la bomba atómica implicó un shock y una pérdida de la inocencia que puso de relieve un severo desafecto en una parte de la comunidad científica hacia la moral e integridad de los científicos (Shapin, 2008). La diferenciación establecida entre la generación de conocimiento verdadero y su utilización tecnológica y social se manifestó abiertamente controvertible, de manera que una parte de la comunidad científica reivindicó ampliar la autorregulación responsable también a las cuestiones relativas al uso y aplicación del conocimiento (Rhodes, 2012). Aquella demanda, que tiene sus raíces en el periodo de entreguerras ante la preocupación generada por la alianza entre la ciencia y la industria militar y en la campaña frente a las armas antinucleares liderada por los científicos, ha tenido reflejo y seguimiento en la revista Bulletin of Atomic Scientists y en el movimiento pugwash auspiciado por Bertrand Russell y Albert Einstein. Ese interés por ampliar la responsabilidad social de los científicos tuvo continuidad en las siguientes generaciones de biólogos moleculares -y científicos ambientalistas y una nueva experticia que opera en las políticas públicas-, tal y como se cristalizó en la conferencia de Asilomar -caso paradigmático de la autogobernanza de la comunidad científica para cuidar las cuestiones emergentes- en respuesta a los riesgos de la investigación en la técnica del ADN recombinante (Barinaga, 2000; Krimsky, 2005).

A lo largo de la segunda mitad del siglo XX la responsabilidad científico-tecnológica se interesó de forma gradual por los problemas ligados a sus propios avances y dinámicas. No obstante, una paradoja que encubren aquellas dinámicas responsables es que la ciencia se concibe como una fuente de riesgos ambientales, sanitarios y éticos al tiempo que se valora su rol en la regulación de esos mismos riesgos. En este sentido, se pretende que la verdad y los códigos de la ciencia se trasladen de manera automática y lineal a la esfera política y legal. Es decir, ese hacerse cargo de los científicos de alguna manera vino a reproducir la imagen de speaking truth to power, como una variante nueva y ampliada del modelo demarcacionista entre ciencia y sociedad (Hoppe, 1999).

A aquella responsabilidad ampliada se unirán progresivamente otras medidas reguladoras de la ciencia con el propósito de modular las innovaciones científico-tecnológicas a través de un sistema de alerta temprana y de una serie de normas restrictivas orientadas principalmente a la protección de la naturaleza y de los valores y principios humanos. En los años 1970, inicialmente en los Estados Unidos se institucionalizan las oficinas asesoras de evaluación tecnológica y las agencias reguladoras que condujeron a las primeras disposiciones legales provistas de información científica sobre los efectos adversos de las tecnologías. Estas políticas reguladoras de la ciencia, reactivas en su origen y que contraponían la ciencia a la sociedad y a la naturaleza, tomaron una orientación probabilística y economicista a través del análisis del riesgo que, adoptando la forma del análisis de riesgo-coste-beneficio, se convirtió en la modalidad de evaluación de tecnologías predominante (López Cerezo y Luján, 2000). Así, los códigos morales internos de la ciencia amplían su responsabilidad a cuestiones que envuelven el riesgo, la seguridad y la protección. De esta manera, la regulación de la actividad científico-tecnológica se centró en la gestión de la I+D per se, que incluía sin contradicción posible la valoración de los riesgos objetivamente delimitados, y una serie de supuestos de competitividad, bienestar y progreso mantenidos al margen de lo cuestionable (Irwin y Wynne, 1995).

En otras palabras, lo relativo al uso y regulación de las consecuencias sociales, ambientales y sanitarias también se circunscribe a cuestiones meramente cognitivas y técnicas propiamente adscritas a la comunidad científica, que de manera progresiva va alcanzando la arena política en su labor de asesoría (Wynne, 1982). Otro fenómeno un tanto paradójico es que la modulación social de las innovaciones científico-tecnológicas mantiene impermeables al debate los objetivos que hay que perseguir, a la vez que una asunción subyacente presupone y legitima la compatibilidad de base entre el crecimiento económico de naturaleza tecnológica e industrial y la sostenibilidad humana y ambiental (Lash, Szerszynski y Wynne, 1996).

Los problemas de tipo epistemológico, normativo y social referidos al análisis del riesgo generaron una respuesta en algunos países europeos que trataban de hacer converger ciencia, tecnología y sociedad, a la vez que a los criterios de factibilidad, eficiencia y fiabilidad del desarrollo científico-tecnológico se les superponían valores como la deseabilidad y el control social. Este modelo constructivo de evaluación de tecnologías que ampliaba el concepto de experticia al ámbito de lo social y reconocía su rol como mediador entre dos mundos tradicionalmente separados sirvió para entrever y anticipar los problemas de fondo acerca de la formulación original de las políticas de I+D, que corresponden a la imagen demarcacionista de la ciencia. 


\section{CIENCIA EN SOCIEDAD: INTEGRACIÓN DE ASPEC- TOS SOCIALES}

A lo largo de las dos últimas décadas la mayor permeabilidad de la ciencia ha venido acompañada de nuevas tensiones entre las dinámicas de apertura y cierre que empañan las fronteras tradicionales entre ciencia y sociedad. Este apartado distingue los diferentes patrones de investigación concurrentes que se despliegan a partir de los años 90 en la formulación de prioridades en las políticas de I+D europeas.

\subsection{La instrumentalización económica de la ciencia}

A lo largo de la última década del siglo XX y de la primera del siglo $\mathrm{XXI}$, las políticas de I+D estuvieron focalizadas en la dimensión vertical, es decir, los programas estratégicos se diseñaban en orden a la mejora y eficiencia de la organización y funcionamiento del sistema de I+D. No obstante, este propósito generalizado por la mejora e integración funcional de los distintos actores del sistema de I+D (apertura), y la omisión de la dimensión horizontal -la discusión sobre los objetivos que hay que perseguir-, si bien relegaron el debate sobre los motivos y la orientación de la investigación científico-tecnológica (cierre), no indican ausencia de valores y propósitos. Al contrario.

La mejora continua en los mecanismos de gobernanza de los sistemas de I+D estuvo principalmente orientada a modernizar y potenciar la capacidad innovadora y competitiva de las empresas -a través de los modelos de la triple hélice y de la cuádruple hélice-, a las cuales parecían estar subordinadas las administraciones públicas y los distintos organismos de I+D (Marklund, Vonortas y Wessner, 2009). Aspectos tan dispares como los planes estratégicos, la movilización de los recursos y los indicadores de evaluación en el conjunto de los agentes involucrados en el conocimiento, la transferencia y la aplicación tecnológica se vieron afectados por el interés cuasiexclusivo del impacto económico de las actividades de I+D (Berman, 2012).

Una de las muchas ironías de este periodo puede resumirse en esta idea: a la vez que se reconocen la naturaleza compleja de la actividad científico-tecnológica y una división del trabajo más interactiva entre los distintos actores del sistema de I+D (se asume la inoperancia del modelo demarcacionista), la diversidad de trayectorias por las que la I+D puede desplegarse y progresar queda seriamente deslucida (la apertura de la actividad científico-tecnológica a la economía achica el espacio de lo posible). A ello se debe que las propiedades normativas de la trayectoria socio- tecnológica en áreas como la energía, el transporte, la agricultura, la salud y la alimentación se hayan decidido sobre un conjunto limitado de intereses y preferencias sociales (Leach, Scoones y Wynne, 2005).

Esta fuerte subordinación de la I+D a las demandas de la macroeconomía y una asunción de facto del carácter inherentemente positivo del progreso se cristalizó en la Estrategia de Lisboa (véase Lisbon European Council 23 and 24 march 2000. Presidency Conclusions). En los años 2000, si bien con muchas dificultades (véase Creating an Innovative Europe), las políticas comunitarias formulan directrices para intensificar la competencia en todos los sectores y adaptar las estructuras y ámbitos sociales -se crean infraestructuras de conocimiento, hay una amplia reforma económica y de modernización del bienestar social y de los sistemas educativos- en respuesta a los retos de la competitividad y la sociedad del conocimiento (véase Commission of the European Communities, 2003).

\subsection{Los aspectos éticos, legales y sociales de la acti- vidad científico-tecnológica}

Las altas expectativas con las que se dotan la ciencia y la tecnología, empero, también han lidiado con las controversias que genera ese mismo poder en la naturaleza y en la sociedad (Jasanoff, 2012). Las distintas controversias originadas durante los años 1990 sobre todo por una serie de crisis alimentarias provocaron una revisión institucional de la manera de considerar y gestionar las implicaciones sociales de la ciencia y la tecnología. En parte por ello, la gobernanza del sistema de I+D se ha robustecido por una incorporación explícita de actividades y fondos de financiación para considerar los aspectos éticos, legales y sociales (Ethical, Legal and Social Aspects Research, ELSA) de la actividad científico-tecnológica. En este sentido, los Programas Marco de la Unión Europea introducen la etiqueta ELSA, que a medio plazo abrió -como sugieren Zwart, Landeweerd y van Rooij (2014)- una senda alternativa a la bioética y se distinguió del programa ELSI (Ethical, Legal and Social Implications Research Program), e impulsan una mayor colaboración e interacción entre expertos de áreas diversas como la de humanidades y ciencias sociales y la de ciencias de la vida (Braun, Herrmann, Konninger y Moore, 2010). ${ }^{1}$

Así, en un periodo corto de tiempo, el enfoque ELSA ha agudizado la necesidad de ampliar los ámbitos de aplicación y de abrir el enmarque de las cuestiones abordables. De esta manera, una primera escalada afecta a la ampliación de ELSA a los programas y áreas de las ciencias de la vida (tercer y cuarto Programa 
Marco, años 1990-1994 y 1994-1998 respectivamente; en el cuarto Programa Marco suponen el $2 \%$ de los fondos asignados a las ciencias y tecnologías de la vida), mientras que a partir del quinto Programa Marco (1998-2002) y sobre todo del sexto Programa Marco (2002-2006) la aplicación de la reflexión social, ética y legal de la actividad investigadora se amplía a todas las áreas del conocimiento. ${ }^{2}$

Por otra parte, la comprensión de la investigación socio-ética varía de manera singular, transita desde su concepción como mero ejercicio externo sobre cuestiones previamente formateadas por los programas científico-ingenieriles a los que acompañaba, cuyo propósito sería la legitimación de las prácticas y agendas de investigación (se esperaba de los investigadores, sobre una clara división del trabajo, una respuesta a las cuestiones que la bioética delimitaba sobre la autonomía personal, el daño o el riesgo), hasta una conceptualización más interactiva -incluyendo actores extraacadémicos-, e integra las cuestiones sociales y económicas abiertas a debate en las propias dinámicas de la I+D (un reflejo parcial de esta transformación epistemológica y metodológica se observa en el séptimo Programa Marco, 2007-2011). Así, la articulación socio-técnica de las actividades de $I+D$, de acuerdo con las narrativas que movilizan los Programa Marco y la financiación de los recursos, se inclinan paulatinamente hacia nuevos patrones de investigación caracterizados por la anticipación -se pone el foco en la definición de la agenda y en la fase de diseño de la trayectoria de la investigación-, la apertura -una interacción mayor con un conjunto más amplio de organizaciones y entidades- y la interdisciplinariedad -se integran cuestiones filosóficas, sociales, económicas y demás en el núcleo de la propia investigación científico-tecnológica (Hilgartner, Prainsack y Hurlbut, 2016). Dicho de otra manera, la integración socio-técnica, originalmente formulada para las ciencias de la vida, se ha ampliado y mejorado progresivamente, tal y como se advierte en la evolución en relación con el ámbito de áreas de conocimiento afectadas, con el conjunto de problemas considerados relevantes, con el tipo de actores involucrados, o con el peso y el grado de influencia sobre los propios procesos de constitución del conocimiento científicotecnológico (von Schomberg, 2007).

Esta progresiva articulación proactiva de áreas, problemas o consideraciones y actores como parte constitutiva de la investigación científico-tecnológica adquiere mayor interés y visibilidad a raíz de la resistencia pública a la comercialización de la biotecnología agroalimentaria y a la inoperancia de los supuestos y mecanismos de regulación a la hora de resolver y responder satisfactoriamente a las controversias sociales en torno a las innovaciones científico-tecnológicas (Levidow, 2009). Aquellas controversias generaron un amplio conjunto de aprendizajes, y las más recientes tecnologías emergentes y facilitadoras -el caso más vistoso es el de las nanotecnologías- han tratado de anticipar una mayor inclusión, reflexividad y deliberación desde las fases iniciales de la investigación (Jasanoff, 2016).

\subsection{La ciencia como elemento capaz de solventar los grandes desafíos}

En los años 2000, a esas dificultades por innovar la gobernanza de la I+D y ampliar actores y demandas en las propias dinámicas de la actividad científicotecnológica, que debe lidiar con una vieja cultura de la gobernanza como mera satisfacción reactiva de una serie de normas limitantes, se une una nueva narrativa instrumentalizadora del conocimiento científicotecnológico.

Más concretamente, las distintas iniciativas e instituciones de I+D empiezan a reconocer ampliamente que la disposición exclusiva a detectar y mejorar las deficiencias de los sistemas de I+D resulta insuficiente e inoperante. Por una parte, adquiere mayor énfasis la idea de que las políticas de I+D, más allá de meras estrategias facilitadoras, exigen una mejor alineación entre políticas de oferta en investigación y tecnología y políticas de demanda, y se plantea maximizar el impacto social y económico de la investigación en diversas áreas, como son las políticas sociales, ambientales, industriales y agrícolas, tal y como se desprende por ejemplo de la estrategia Europa 2020 (véase European Commission, 2010). En este mismo sentido, un destacado informe de expertos sobre la razón de ser del Espacio Europeo de Investigación indica:

Nuestro argumento central es que, para avanzar, ERA [European Research Area] necesita equilibrar su enfoque actual en la estructura y el proceso con un mayor énfasis en el contenido y los productos. La motivación para las reformas e inversiones demandadas por un ambiente amigable solo vendrá cuando tal asociación se haga" (European Commission, 2008, p. 36).

Por otra parte, empieza a alcanzar solidez narrativa la idea de que las políticas de I+D deben formularse como "capaces de solventar problemas" o de ser partícipes en la respuesta a una serie de retos que afectan a nuestras sociedades. Este mayor énfasis en los resultados de la actividad científico-tecnológica 
pronto se traduce en una serie de grandes desafíos que definen las prioridades de investigación europeas (salud, sociedad inclusiva y segura, industria, clima, energía y movilidad, y alimentos y recursos naturales) y, como se declara oficialmente en la Declaración de Lund (2009), para ello deben subvertirse las fronteras disciplinares de investigación y desplazar los patrones de investigación colaborativos a los retos sociales. ${ }^{3}$

Esto viene a sugerir que la naturaleza y la responsabilidad de las actividades de I+D, que amplían su radio de acción desde las dimensiones estrictamente procedimentales hasta otras más sustantivas que afectan a los objetivos y a los resultados perseguidos, se determinan progresivamente en función de su disponibilidad y efectividad para mejorar realidades "ajenas" a sí mismas. Más concretamente, se establecen una serie de retos o desafíos que inclinan y dirigen el conjunto de trayectorias por las que la investigación científicotecnológica puede desarrollarse y progresar.

\section{LA INVESTIGACIÓN E INNOVACIÓN RESPONSABLES}

El más reciente Programa Marco de Investigación de la Unión Europea, Horizon 2020 (2014-2020) (véase European Commission, 2011), tiene una serie de particularidades, entre las cuales puede destacarse el hecho de que la investigación e innovación responsables (Responsible Research and Innovation, RRI por sus siglas en inglés) se convierte en una parte integral de la financiación de la investigación europea. Más concretamente, Horizon 2020 tiene como objetivo general "contribuir a la construcción de una sociedad y una economía basadas en el conocimiento y la innovación". Para ello fija tres prioridades: "generar una ciencia excelente", "fomentar el liderazgo industrial para apoyar a las empresas" y "afrontar los retos de la sociedad para aportar una respuesta directa a los retos enumerados en la estrategia Europa 2020", y establece dos objetivos específicos: "difundir la participación y ampliar la excelencia" y "ciencia con y para la sociedad". Una de sus particularidades es que señala quince temas transversales, uno de los cuales es la RRI.

La emergencia de la RRI, que sigue la senda progresiva de transformar los patrones de investigación y moviliza a distintos actores, intereses y necesidades, adquiere sentido a la luz de la innovación gradual de los roles y de las responsabilidades en la investigación científico-tecnológica que estamos viendo a lo largo de este artículo. Uno de los primeros informes sobre la RRI, redactado por Sutcliffe con los materiales de debate recopilados en el workshop del departamento de Investigación e Innovación de la Comi- sión Europea sobre RRI en Europa (véase A Report on Responsible Research \& Innovation), registraba cinco principales debates: "un enfoque deliberado sobre la investigación y la innovación para alcanzar beneficio social y ambiental", "una participación social amplia e inclusiva a lo largo de todo el proceso de innovación", "una evaluación de los riesgos y las oportunidades sociales, éticas y ambientales futuros, simultáneos a los técnicos y comerciales", "mecanismos de anticipación para gobernar los retos y beneficios y que posibiliten la adaptabilidad y repuesta a los cambios en el conocimiento y las circunstancias" y "la apertura y la transparencia como ingredientes constitutivos de los procesos de investigación e innovación". ${ }^{4}$ Sutcliffe (véase A Report in Responsible Research \& Innovation, p. 5) precisaba:

RRI trata de mejorar para anticiparse a los problemas, teniendo en cuenta cuestiones sociales, éticas y ambientales más amplias y poder crear sistemas flexibles y adaptables para hacer frente a estas consecuencias no deseadas. Esto a veces se llama "gobernanza anticipatoria".

La gobernanza anticipatoria indica la importancia de sobreponerse al lenguaje determinista de las políticas de desarrollo científico-tecnológico y se singulariza por la capacidad de "gobernar la emergencia de tecnologías basadas en el conocimiento mientras su gestión todavía es aún posible" (Guston, 2014 , p. 128). Así, la anticipación también difiere de la predicción e incorpora en su seno la inclusión de conocimientos, valores y demandas cuya deliberación escruta los objetivos sociales a los que la investigación y la innovación científico-tecnológica deben ofrecer respuesta. Como aclaran Stilgoe y Guston (2017), “la cuestión no es preguntar qué pueden hacer las tecnologías emergentes para ayudarnos, sino más bien formular la pregunta previa sobre qué podemos hacer para ayudar, o no, a las tecnologías emergentes" (p. 857).

Por su parte, von Schomberg propone impulsar la investigación y la innovación científico-tecnológica hacia los "impactos adecuados", anclados en los valores articulados en el Tratado de la Unión Europea, y define la RRI como "un proceso transparente e interactivo mediante el cual los actores sociales y los innovadores se vuelven mutuamente receptivos, con vistas a la aceptabilidad (ética), la sostenibilidad y la deseabilidad social del proceso de innovación y de sus productos comercializables para permitir una adecuada integración de los avances científicos y tecnológicos en nuestra sociedad" (von Schomberg, 2013, p. 9). 
Esta definición sintetiza los principales rasgos emergentes de la RRI (Owen, Macnaghten y Stilgoe, 2012): abre a discusión los propósitos más amplios de la actividad científico-tecnológica, institucionaliza los mecanismos de corresponsabilidad entre los distintos actores, y reenmarca la responsabilidad en respuesta a un contexto en el que la naturaleza distribuida del conocimiento y la creciente incertidumbre e imprevisibilidad menguan principios meramente formalistas y consecuencialistas (Adam y Groves, 2011).

En este sentido, la RRI es un concepto muy vago que tiene como propósitos sincrónicos una adhesión amplia -de ahí en parte su ideario generalista- y la suficiente concreción para su operacionalización razonable (Rip, 2016). ${ }^{5}$ A su vez, el sentido y la finalidad de la RRI manifiestan una gobernanza proactiva que -más allá de la mitigación de las consecuencias adversas de la I+D y de su valor de mercado- despliega la diversidad de expectativas y capacidades para generar futuros plausibles y deseados (Ribeiro, Smith y Millar, 2017); la RRI apela a desplazar el foco a las preferencias y a los valores que sustentan la I+D. Unido a ello, la RRI se ensambla con la significación de los "grandes desafíos", desplaza el producto y la finalidad de la investigación desde el conocimiento y la curiosidad a los retos sociales, por lo que excede la utilidad puramente económica, y reitera los límites de incrementar los impactos a través de políticas que acentúan exclusivamente el lado de la oferta de la I+D (ver apartado 3.3). Otra peculiaridad de la RRI es que transciende los roles establecidos en la investigación científica y su relación con la sociedad en aras de una comprensión epistémica y social más abierta y relacional de la investigación y de la innovación científico-tecnológicas. ${ }^{6}$

De alguna manera, la RRI insta simultáneamente a resultados e impactos responsables y a procesos responsables. No obstante, estas consideraciones están sujetas a riesgo si no incorporan la autocrítica sobre los límites, las tensiones y las posibilidades de la propia RRI. No menos importante es recordar que la institucionalización de la RRI encuentra dificultades, resistencias y limitaciones por las trayectorias dependientes que condicionan fuertemente las potencialidades de la propia RRI a las decisiones tomadas en el pasado y a una visión primariamente económica de la I+D (apartados 3.1. y 3.3.). Una gobernanza democrática de la I+D encuentra una serie de limitaciones que constituyen el contenido, la evolución y el alcance de las políticas responsables de ciencia y tecnología en Europa:
- Los actores involucrados en la deliberación tienen intereses y demandas heterogéneos, es decir, una amplia variedad de posibles escenarios ligados a la I+D, que en buena medida dilucidan los contornos de la negociación (Saille, 2015; van Oudheusden, 2014). En este sentido, resulta ingenuo pensar que un procedimiento responsable (abierto, participativo, inclusivo) va a contribuir automáticamente al logro de unos objetivos previamente establecidos (i.e., los impactos justos de von Schomberg). Por el contrario, un procedimiento abierto que implica distintas perspectivas e intereses puede robustecer las decisiones y mejorar su calidad epistémica.

- Las normas que rigen la deliberación y los objetivos que hay que perseguir están sujetas a discusión, por lo que la apelación ética puede condicionar la naturaleza política de las controversias. De igual modo, la reducción de los impactos justos a una serie de valores previamente determinados y la apelación al compromiso ético sobre la base de unos procedimientos deliberativos desplazan la función esencial del desacuerdo y la contestación en la generación de vínculos de corresponsabilidad entre actores implicados.

- La ética de la investigación tiene otras limitaciones (Rip, 2014), como un alcance práctico limitado (códigos de conducta y procedimientos de consentimiento informado) y una interpretación utilitaria y consecuencialista de la responsabilidad (maximizar las contribuciones positivas de la tecnología y minimizar los posibles resultados negativos). ${ }^{7}$

\section{CONSIDERACIONES FINALES}

En este artículo hemos visto que las políticas de I+D tienen un carácter histórico y la imagen que relaciona automáticamente ciencia, progreso y responsabilidad ha dependido de un conjunto singular de factores analíticos y contextuales. Esto hace que el modelo lineal de I+D y la capacidad autorreguladora de los científicos hayan sido revisados y mejorados con arreglo a una serie de evidencias y demandas históricas. En este sentido, la demarcación ciencia-sociedad que ha regido las políticas de I+D se ha sostenido por una tensión irresoluble entre la autonomía de la ciencia y una demanda creciente de responsabilidad social y rendición de cuentas. 
Ello no obstante, las asunciones que subyacen al criterio demarcacionista nos alertan de su valor simbólico y retórico en las políticas de I+D. A ello se unen otros hechos históricos: una clara división de roles entre los actores; la supeditación de la actividad científico-tecnológica a las demandas macro-económicas; una identificación por defecto de la investigación con el progreso y el bienestar. Sin embargo, este artículo ha sostenido la emergencia de nuevas formas de producción de conocimiento que lidian con aquellos patrones de investigación basados en disciplinas y áreas de conocimiento. A su vez, hay una mayor sensibilidad hacia el entorno y una creciente orientación social y económica de la I+D que acentúa el carácter distribuido y colectivo del conocimiento.
Este artículo ha arrojado luz sobre esas dinámicas de apertura y cierre: se observa una clara evolución hacia una comprensión interactiva de la ciencia y de sus relaciones con la sociedad, pero no carente de resistencias y tensiones, en parte por el impulso prescriptivo de la I+D y su comprensión como un ingrediente primariamente económico, que merma las posibilidades de discusión, lo que explica por qué algunas de las relaciones y demandas indicadas en este artículo se muestran más resistentes al cambio. Ese acomodo conflictivo entre objetivos es lo que viene dando forma a las políticas europeas de investigación científico-tecnológica.

\section{NOTAS}

1. El programa ELSI se establece con e Programa Genoma Humano a inicios de 1990 en los Estados Unidos y tenía como misión anticipar las implicaciones éticas, legales y sociales de la genética y la genómica, mientras que el enfoque ELSA reconoce desde sus orígenes la necesidad de ampliar su ámbito de investigación y desbordar la naturaleza lineal que representa el estudio de las implicaciones (Zwart y Nelis, 2009; Zwart, Landeweerd y van Rooij (2014). No obstante, hay una diversidad y heterogeneidad de perspectivas y métodos sobre la práctica de la ética en las actividades científico-tecnológicas (véase Reijers et al., 2017).

2. En el sexto Programa Marco a través de las distintas prioridades y acciones la Comisión Europea financió veinte proyectos en el ámbito de ELSA y gobernanza de la nanotecnología (Hullmann, 2008).

\section{BIBLIOGRAFÍA}

Adam, B. y Groves, G. (2011). Futures tended: Care and future-oriented responsibility. Bulletin of Science, Technology \& Society, 31 (1), pp. 17-27. https://doi. org/10.1177/0270467610391237

Barinaga, M. (2000). Asilomar Revisited: Lessons for Today? Science, 287 (5458), pp. 1584-1585. https://doi. org/10.1126/science.287.5458.1584

Berman, E. P. (2012). Creating the Market University: How Academic Science Became an Economic Engine. Princeton: Princeton University Press. https://doi. org/10.1515/9781400840472
3. Con ese propósito general, a la Declaración de Lund (2009) se han unido la Declaración de Vilnius sobre Horizontes para las ciencias sociales y las humanidades (2013) y la Declaración de Roma sobre Investigación e Innovación Responsables en Europa (2014).

4. Una propuesta general sobre un marco de trabajo (dimensiones, mecanismos) en RRI puede encontrarse en Stilgoe, Owen y Macnaghtell, 2013 y en Owen, Stilgoe, Macnaghtell, Gorman, Fisher y Guston, 2013

5. Muestra de ello es la ambigüedad sobre la naturaleza, el propósito y el alcance de la RRI que puede descubrirse entre los distintos informes de la Comunidad Europea (véanse European Commission, 2012; European Commission, 2013; European Commission, 2014). En cualquier caso, la RRI también debe comprenderse -más allá de las diná-

Braun, K., Herrmann, S. L., Konninger, S. and Moore, A. (2010). Ethical Reflection Must Always Be Measured. Science, Technology \& Human Values, 35 (6), pp. 839-864. https://doi. org/10.1177/0162243909357917

Commission of the European Communities (2001). European Governance: A White Paper. [En línea]. Disponible en https:// ec.europa.eu/europeaid/sites/devco/files/communication-white-paper-governance-com2001428-20010725_en.pdf

Commission of the European Communities (2003). Communication from the Commission to the Council and the European micas tipo top-down que se diseñan y ejecutan en planes comunitarios- como una serie de iniciativas que se generan en la sociedad civil y se robustecen con los programas institucionales. Véase a este propósito la iniciativa Nanojury (Singh, 2008).

6. Para una visión panorámica sobre el recorrido que viene realizando la RRI (organizaciones más relevantes, proyectos, áreas de aplicación, etc.) véase Timmermans (2017). Una valoración sobre el alcance y la relevancia de la RRI puede verse en European Commission (2018).

7. Dicho de otra manera, una interpretación reduccionista puede llegar a ser uno de los principales obstáculos para desempeñar las potencialidades de una perspectiva de RRI (Eizagirre, Rodríguez e Ibarra, 2017; Levidow y Neubauer, 2014).

Parliament. Some Key Issues in Europe's Competitiveness - Towards an Integrated Approach. [En línea]. Disponible en https://ec.europa.eu/transparency/ regdoc/rep/1/2003/EN/1-2003-704EN-F1-1.Pdf

Douglas, H. E. (2003). The Moral Responsibilities of Scientists (Tensions Between Autonomy and Responsibility). American Philosophical Quarterly, 40 (1), pp. 59-68.

Eizagirre, A., Rodríguez, H. e Ibarra, A. (2017). Politicizing Responsible Innovation: Responsibility as Inclusive Governance. International Journal of Innova- 
tion Studies, 1 (1), pp. 20-36. https:// doi.org/10.3724/SP.J.1440.101003

Elzinga, A. (2012). Features of the Current Science Policy Regime: Viewed in Historical Perspective. Science and Public Policy, 39 (4), pp. 416-428. https://doi. org/10.1093/scipol/scs046

European Commission (2002). Science and Society. Action Plan. Luxembourg: Office for Official Publications of the European Communities. [En línea]. Disponible en https://ec.europa.eu/research/swafs/ pdf/pub_gender_equality/ss_ap_en.pdf

European Commission (2007). Taking European Knowledge Society Seriously. Report of the Expert Group on Science and Governance to the Science, Economy and Society Directorate. Luxembourg: Office for Official Publications of the European Communities.

European Commission (2008). Challenging Europe's Research: Rationales for the European Research Area (ERA). Report of the ERA Expert Group. Luxemburg: Office for Official Publications of the European Communities.

European Commission (2010). Communication from the Commission. Europe 2020. A Strategy for Smart, Sustainable and Inclusive Growth. [En línea]. Disponible en https://ec.europa.eu/eu2020/pdf/ COMPLET\%20EN\%2OBARROSO\%20\%20 $\% 20007 \% 20$-\%20Europe $\% 202020 \% 20$ -\%20EN\%20version.pdf

European Commission (2011). Communication from the Commission to the European Parliament, the Council, the European Economic and Social Committee and the Committee of the Regions. Horizon 2020 - The Framework Programme for Research and Innovation. [En línea]. Disponible en https:// ec.europa.eu/research/horizon2020/ pdf/proposals/communication_from_ the_commission_-_horizon_2020_-the_framework_programme_for_research_and_innovation.pdf

European Commission (2012). Ethical and Regulatory Challenges to Science and Research Policy at the Global Level. Luxemburg: Office for Official Publications of the European Communities. [En línea]. Disponible en http://ec.europa. eu/research/science-society/document_library/pdf_06/ethical-and-regulatory-challenges-042012_en.pdf

European Commission (2013). Options for Strengthening. Responsible Research and Innovation Report of the Expert Group on the State of Art in Europe on Responsible Research and Innovation. Luxemburg: Office for Official Publications of the European Communities. [En línea]. Disponible en https://ec.europa. eu/research/science-society/document_library/pdf_06/options-for-strengthening_en.pdf

European Commission (2014). Responsible Research and Innovation: Europe's Ability to Respond to Societal Challenges. Publications Office of the European Union. [En línea]. Disponible en https:// ec.europa.eu/research/swafs/pdf/pub_ rri/KI0214595ENC.pdf

European Commission (2018). Monitoring the Evolution and Benefits of Responsible Research and Innovation in Europe. Luxemburg: Office for Official Publications of the European Communities. [En línea]. Disponible en https://publications. europa.eu/en/publication-detail/-/ publication/2c5a0fb6-c070-11e89893-01aa75ed71a1/language-en

Flink, T. y Kaldewey, D. (2018). The New Production of Legitimacy: STI Policy Discourses Beyond the Contract Metaphor. Research Policy, 47 (1), pp. 14-22. https://doi.org/10.1016/j.respol.2017.09.008

Guston, D. H. (2014). Understanding 'Anticipatory Governance'. Social Studies of Science, 44 (2), pp. 218-242. https://doi. org/10.1177/0306312713508669

Hilgartner, S., Prainsack, B. y Hurlbut, J-B. (2016). Ethics as Governance in Genomics and Beyond. En: Felt, U., Fouché, R., Miller, C. A. y Smith-Doerr, L. (eds). The Handbook of Science and Technology Studies. Cambridge, MA: The MIT Press, pp. 823-851.

Hoppe, R. (1999). Policy Analysis, Science and Politics: From 'speaking truth to power' to 'making sense together'. Science and Public Policy, 26 (3), pp. 201-210. https://doi. org/10.3152/147154399781782482

Hullmann, A. (2008). European Activities in the Field of Ethical, Legal and Social Aspects (ELSA) and Governance of $\mathrm{Na}$ notechnology. European Commission, DG Research, Unit "Nano and Converging Sciences and Technologies". [En línea]. Disponible en https://www.nanowerk.com/nanotechnology/reports/ reportpdf/report122.pdf
Irwin, A. y Wynne, B. (eds.) (1995). Misunderstanding Science? The Public Reconstruction of Science and Technology. Cambridge, UK: Cambridge University Press. https://doi.org/10.1017/ CB09780511563737

Jamison, A. (2012). Science and Technology in Postwar Europe. En: Stone, D. (ed.). The Oxford Handbook of Postwar European History. Oxford: Oxford University Press, pp. 630648. https://doi.org/10.1093/oxfordhb/9780199560981.013.0032

Jasanoff, S. (2012). Science and Public Reason. London: Routledge. https://doi. org/10.4324/9780203113820

Jasanoff, S. (2016). The Ethics of Invention: Technology and the Human Future. New York: Norton.

Kaldewey, D. y Schauz, D. (eds.). (2018). Basic and Applied Research: The Language of Science Policy in the Twentieth Century. New York. Berghahn Books. https://doi.org/10.2307/j.ctv8bt0z7

Kleinman, D-L. (1995). Politics on the Endless Frontier: Postwar Research Policy in the United States. Durham: Duke University Press.

Krismky, S. (2005). From Asilomar to Industrial Biotechnology: Risks, Reductionism and Regulation. Science as Culture, 14 (4), pp. 309-323. https://doi. org/10.1080/09505430500368998

Lash, S., Szerszynski, B. y Wynne, B. (1996). Risk, Environment and Modernity: Towards a New Ecology. London: Sage.

Leach, M., Scoones, I. y Wynne, B. (eds.) (2005). Science and Citizens. Globalization and the Challenge of Engagement. London: Zed Books.

Levidow, L. (2009). Democratizing agri-biotechnology? European public participation in agbiotech assessment. Comparative Sociology, 8(4), pp.541-564. https:// doi.org/10.1163/156913309X461633

Levidow, L. y Neubauer, C. (2014). EU Research Agendas: Embedding What Future? Science as Culture, 23 (3), pp. 397412. https://doi.org/10.1080/0950543 1.2014 .926149

López Cerezo, J. A. y Luján, J. L. (2000). Ciencia y política del riesgo. Madrid: Alianza.

Marklund, G., Vonortas, N. y Wessner, Ch. (eds.). (2009). The Innovation Imperative: National Innovation Strategies in the Global Economy. Chelten- 
ham. UK: Edward Elgar. https://doi org/10.4337/9781848446090

Merton, R. (1942). A Note on Science and Democracy. Journal of Legal and Political Sociology, 1, pp. 115-126.

Nelson, R. R. y Wright, G. (1992). The Rise and Fall of American Technological Leadership: The Postwar Era in Historical Perspective. Journal of Economic Literature, 30 (4), pp. 1931-1964.

Oudheusden, M. van (2014). Where are the politics in responsible innovation? European governance, technology assessments, and beyond. Journal of Responsible Innovation, 1 (1), pp. 67-86. https://doi.org/10.1080/2 3299460.2014.882097

Owen, R., Macnaghten, P. y Stilgoe, J. (2012). Responsible research and innovation: from science in society to science for society, with society. Science and Public Policy, 39 (6), pp. 751-760. https://doi.org/10.1093/scipol/scs093

Owen, R., Stilgoe, J., Macnaghtell, P., Gorman, M., Fisher, E. y Guston, D. (2013). A Framework for Responsible Innovation. En: Owen, R., Bessant, J. y Heintz, M. (eds.). Responsible Innovation. Managing the Responsible Emergence of Science and Innovation in Society. Chichester: Wiley, pp. 27-50. https://doi. org/10.1002/9781118551424.ch2

Polanyi, M. (1951). The Logic of Liberty: Reflections and Rejoinders. Chicago: University of Chicago Press.

Reijers, W., Wright, D., Brey, P., Weber, K., Rodrigues, R., O'Sullivan, D. y Gordijn, B. (2017). Methods for Practising Ethics in Research and Innovation: A Literature Review, Critical Analysis and Recommendations. Science and Engineering Ethics, 24 (5), pp. 1437-1481. https:// doi.org/10.1007/s11948-017-9961-8

Rhodes, R. (2012). The Making of the Atomic Bomb. New York: Simon \& Schuster.

Ribeiro, B. E., Smith, R. D. J. y Millar, K. (2017). A mobilising concept? Unpacking academic representations of responsible research and innovation. Science and Engineering Ethics, 23 (1), pp. 81-103. https://doi.org/10.1007/ s11948-016-9761-6

Rip, A. (2014). The Past and Future of RRI. Life Sciences, Society and Policy, 1 (10), pp. 1-15. https://doi.org/10.1186/ s40504-014-0017-4

Rip, A. (2016). The Clothes of the Emperor: An Essay on RRI in and around Brussels. Journal of Responsible Innovation, 3 (3), pp. 290-304. https://doi.org/10.1080/2 3299460.2016.1255701

Saille, S. de (2015). Innovating Innovation Policy: The Emergence of 'Responsible Research and Innovation'. Journal of Responsible Innovation, 2 (2), pp. 152168. https://doi.org/10.1080/2329946 0.2015 .1045280

Sarewitz, D. (1996). Frontiers of Illusion: Science, Technology and the Politics of Progress. Philadelphia: Temple of University Press.

Sarewitz, D. (2016). Saving Science. The New Atlantis, 49, pp. 4-40.

Schomberg, R. von (2007). From the Ethics of Technology Towards an Ethics of Knowledge Policy \& Knowledge Assessment. A working document from the European Commission Services. [En línea]. Disponible en https://publications.europa.eu/en/publicationdetail/-/publication/aa44eb61-5be243d6-b528-07688fb5bd5a

Schomberg, R. von (2013). A Vision on Responsible Research and Innovation. En: Owen, R., Bessant, J. y Heintz, M. (eds.). Responsible Innovation. Managing the Responsible Emergence of Science and Innovation in Society. Chichester: Wiley, pp. 51-74. https://doi. org/10.1002/9781118551424.ch3

Shapin, S. (2008). The Scientific Life: A Moral History of a Late Modern Vocation. Chicago: The University of Chicago Press. https://doi.org/10.7208/chicago/9780226750170.001.0001

Singh, J. (2008). The UK Nanojury as 'upstream' public engagement. Participatory Learning and Action, 58 (1), pp. 27-32.

Steelman, J. R. (1948). Science and Public Policy: A Report by the President's Scientific Research Board. Bulletin of the Atomic Scientists, 4 (1), pp. 23-31. https://doi.org/10.1080/00963402.194 8.11460154

Stilgoe, J. y Guston, D. (2017). Responsible Research and Innovation. En: Felt, U.,
Fouché, R., Miller, C. A. y Smith-Doerr, L. (eds). The Handbook of Science and Technology Studies. Cambridge, MA: The MIT Press, pp. 853-880.

Stilgoe, J., Owen, R. y Macnaghten, P. (2013). Developing a framework for responsible innovation. Research Policy, 42 (9), pp. 1568-1580. https://doi. org/10.1016/j.respol.2013.05.008

Timmermans, J. (2017). Mapping the RRI Landscape: An Overview of Organisations, Projects, Persons, Areas and Topics. En: Asveld, L., Dam-Mieras, R. van, Swierstra, T., Lavrijssen, S., Linse, K. y Hoven, J. van den (eds.). Responsible Innovation 3: A European Agenda? Cham: Springer, pp. 21-47. https://doi. org/10.1007/978-3-319-64834-7_3

Wynne, B. (1982). Institutional Mythologies and Dual Societies in the Management of Risk. En: Kunreuther, H. C. y Ley, E. V. (eds.). The Risk Analysis Controversy: An Institutional Perspective. Berlin: Springer Verlag, pp. 127-143. https://doi. org/10.1007/978-3-642-81940-7_10

Zwart, H., Landeweerd, L. y Rooij, A. van (2014). Adapt or perish? Assessing the recent shift in the European research funding arena from 'ELSA' to 'RRI'. Life Sciences, Society and Policy, 10 (11), pp. 1-19. https://doi.org/10.1186/s40504014-0011-x

Zwart, H. y Nelis, A. (2009). What is ELSA genomics? EMBO Reports, 10 (6), pp. 540-544. https://doi.org/10.1038/embor.2009.115

\section{Otros recursos}

A Report on Responsible Research \& Innovation. [En línea]. Disponible en https://ec.europa.eu/research/sciencesociety/document_library/pdf_06/rrireport-hilary-sutcliffe_en.pdf

Creating an Innovative Europe. Report of the Independent Expert Group on R\&D and Innovation appointed following the Hampton Court Summit and chaired by Mr Esko Aho. Luxemburg: Office for Official Publications of the European Communities, 2006.

Lisbon European Council 23 and 24 march 2000. Presidency Conclusions. [En línea]. Disponible en https://www.europarl.europa.eu/summits/lis1_en.htm 